\title{
A Proposal of Linked Data for Smart Metering System
}

\author{
Juan C. Olivares-Rojas, Enrique Reyes-Archundia, and José A. Gutiérrez-Gnecchi \\ Tecnológico Nacional de México / I.T. de Morelia \\ \{juan.or, enrique.ra, jose.gg3\}@morelia.tecnm.mx
}

\begin{abstract}
This paper shows the proposal of the use of linked data for smart metering systems. The aim of linked data in smart grid applications such as smart metering systems is to give semantic knowledge of the energy transactions between all participators in the power markets. In combination with other technologies like blockchain, the linked data could improve decentralization and security in the world's energetic future.
\end{abstract}

Keywords: JSON-LD, Linked Data, Smart Meters, Transactive Energy.

\section{Introduction}

Energy systems are the main engine of human activities; mainly, the electrical energy developed at the end of the 19th century has been a key element in transforming human society. On the other hand, information and communication technologies' maturation is integrated into industrial activities, changing the world we live in and managing it increasingly digital. This digitization has fallen on the electrical power systems, providing them with communication and processing capabilities that provide a specific intelligence to the traditional electrical grid. The smart grid (SG) allows the generation, transmission, distribution, and electrical energy consumption to be made more efficient, making it more environmentally friendly, reliable, and cheaper for end users. One of the smart grid's fundamental areas is smart metering systems (SMS). The electric energy meter called smart meter allows continuous monitoring of electricity consumption and reporting it to the electricity company's facilities without an operator's need. Besides, power outages and reconnections can be dynamically automated. Currently, end-users have stopped being passive consumers of micro electricity generators by using renewable energy sources such as solar panels and wind turbines, which are becoming cheaper; for this reason, smart meters can measure electrical energy production to integrate into the electrical grid. More and more functionalities are being added to smart meters (SM), such as measuring electrical power quality events, integrate with demand response systems, and electrical energy management. The cost of energy can be managed in various schemes such as flat rate, time zone, and recently working prices in real-time.

End-users can view their electrical energy consumption and cost data through web portals and mobile applications. Generally, the data can be displayed graphically, querying historical data and performing data analysis that determines electricity consumption/production patterns. Unfortunately, this data does not store information. Data analytics applications such as energy theft and fraud, fault detection, improvement of power quality, forecast of demand, and energy consumption, among others, are required data with significance.

The data has been found in SM with simple embedded database systems, but as hardware gets cheaper and cheaper, they begin to integrate more robust databases. Generally, the data has stored 
in intermediate devices called data concentrators (DC) that collect the information from a set of smart meters in a given region and then send it to other larger concentrators or directly to the electric company's data centers. The servers in the utility's data centers concentrate a large volume of immense data. The computing architecture at the edge-fog cloud can be integrated into the architecture of smart metering systems. Still, the data has no meaning that allows it to be operated and integrated into other schemes within the applications that smart cities require.

The main contribution of this work is to be useful to a better power market with the use of open linked data, which improves the operation of SG and its technological infrastructure. This work could reduce the rates of electricity with better participation of all the parties in the power market.

\section{State of the art}

The use of semantics in smart grids has been around for several years now, but as of today, it has not yet been fully exploited. Resource Description Framework (RDF) and ontologies have been handled, as well as generic standards such as the Common Information Model (CIM) [1]. Additionally, the use of graphs and other semantic technologies have been applied in different applications included the SG [2].

Several works have been carried out to standardize smart grids data, particularly in smart metering systems, such as Smart Meter Ontology [3] and the OMS ontology [4]; these two works focused on the research part. In the practical part, some consortia, such as the Smart Electric Power Alliance, have focused their efforts on standardizing semantic measures in the Smart Grid Ontology [5]. Given that electrical power systems are critical infrastructures for all nations of the world, governments have taken it upon themselves to standardize and control their use. For this reason, for example, in the United States, the National Institute of Standards and Technology (NIST) has been in charge of defining an open standard for the development of ontologies for the smart grid [6].

Recently, interest in standardizing semantic data for the smart grid has returned [7]. Studies have been carried out to handle semantic data in specific domains such as buildings [8] and the flexibility of electricity demand [9]. Other works are focused in ontologies [10], databases [11], and semantic graphs [12].

Below a comparison of the related works is shown in Table I

Table I. Related Works

\begin{tabular}{|l|l|l|l|}
\hline Work & Semantic Technology & SG/SMS & Power Market \\
\hline$[1]$ & RDF & SG & Billing \\
\hline$[3]$ & Ontology & SMS & \\
\hline$[4]$ & Ontology & SMS & Billing \\
\hline$[5]$ & Ontology & SG & \\
\hline$[6]$ & Ontology & SG & Trading/Commercialization \\
\hline$[7]$ & Ontology & SG & \\
\hline$[8]$ & Ontology & SG & \\
\hline$[9]$ & Ontology & SG & Commercialization \\
\hline$[10]$ & Ontology & SG & \\
\hline$[11]$ & Database & SG & \\
\hline$[11]$ & Database & SG/SMS & \\
\hline
\end{tabular}




\begin{tabular}{|l|l|l|l|}
\hline$[12]$ & Ontology & SG & \\
\hline This work & JSON-LD & SMS & P2P Transactive Energy \\
\hline
\end{tabular}

Despite the existence of several works in charge of defining data in the smart grid, there is a lack of definitions that allow integration to the new data domains of smart grid applications such as transactive energy systems, detection, and location of faults, detection of theft and energy fraud, improvement of energy quality, among others.

For this reason, our proposal consists of analyzing more analytically through experimentation and taking into account other factors such as portability and use of a data set as an ontology in the domain of smart metering systems and adding the definition of terms of missing applications. It is proposed to use Python language to ease use and possible integration into machine learning libraries.

\section{A Linked Data Architecture for Smart Metering Systems}

The most implemented SMS architecture is the Advanced Metering Infrastructure (AMI) [14], which consists of a set of SM, Data Concentrators (DC), DBMS, wired and wireless communication means. The SM is the key component of SMS due to its capabilities to process, store and communicate electrical data. The data has been visualized into Web or mobile applications where the end-users can check their energy consumption and pay it. Besides, the utility can automate some processes such as cuts and reconnection without human operators.

The proposed solution consists of standardizing and using a smart metering system with linked open data. Through a web portal and mobile application, they can connect with other public service companies and applications. It will give meaning to the data and improve the power grid's operation in its IT infrastructure.

Traditionally power markets are focused on big parties: utility operators, generators, transmitters, distributors, commercialists, aggregators, among other participants. With the proliferation of lowercost removable Distributed Energy Resources (DER) like solar panels and wind turbines, the role of end-users has changed from consumers to prosumers (producer and consumer) [15]. One of the new applications of SM is related to handle dynamic prices and rates of energy. If there is a high energy production or offer, the utility can send discount signal prices to their clients with the idea of increment consumption. On the other hand, if there is a lack of energy production, utilities can buy energy from the prosumer with low costs. For these reasons, data in SMS must have significance.

It is intended to use the JSON-LD format where the data once exposed on the Web can be used by third parties. A relevant part is the management of security and privacy schemes that allow having granularity schemes that allow reliable, complete, and available access to the data. One of the most vital trends in information security and privacy is distributed ledger technology schemes such as blockchains. Blockchain systems such as cryptocurrencies allow the decentralization of electrical energy consumption/production transactions, making it ideal for managing transactive energy systems, where prosumers can exchange energy widely. 
The proposed linked data architecture for SMS has shown in Figure 1.

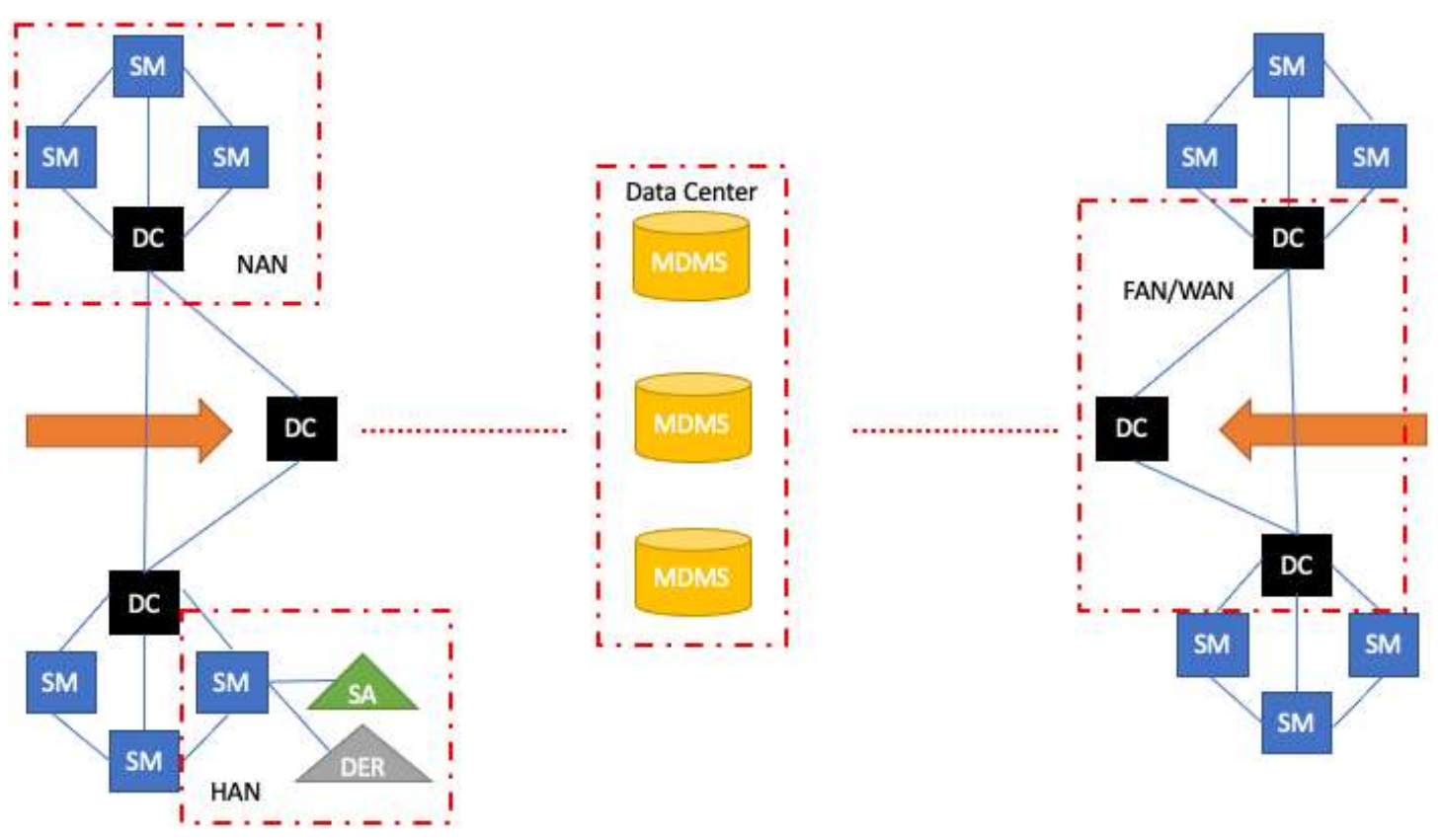

Figure 1. A linked data architecture for smart metering systems.

We can observe that SM is connected to loads as appliances and other electrical devices. Also, SM can be connected with microgeneration (DER). Notably, some loads and devices have intelligent consumption, such as smart appliances (SA). The communication between SM, SA, and I in some cases, DER produce a home area network (HAN) similar to a local area network.

The communication of SM between other SM and DC forms a neighborhood area network (NAN). This network is similar to a campus area network or metropolitan area network, depending on the geographical distance.

The DC can communicate with other DC large distances using the transmission and distribution lines or fiber optics. This last communication is called field area network (FAN) that in essence, is a vast area network with the difference of use rugged devices.

At the headend, the final DCs arrives at the Data Center where the utilities are applications such as metering data management system (MDMS), billing, outages, geographical information systems (GIS).

This architecture must adapt to an edge-fog-cloud architecture where the edge has represented by the HAN devices (SM, DER, SA), the fog layer is composed by NAN/FAN devices (SM, DC), and finally the cloud tier is represented by the servers at the data center.

Bellow, we presented a scenario where prosumers, utilities, and other power market participants can interact. 
Scenario 1: In zone part of Mexico North, there was a snowfall, and some of the generation plants were down. The utility sends signals to other market participants, mainly prosumer in central and south of Mexico, with attractive prices for buying energy surplus. The prosumers through SM can decide if they send their production or store his energy (if they have electrical storage).

Figure 2 shows an example of linked data using JSON-LD in SMS.

\begin{tabular}{|l|l|}
\hline Price signal sent to prosumers & Prosumers response \\
\hline$\left\{\begin{array}{l}\text { "@context": } \\
\text { "http://dsc.morelia.tecnm.mx/smste", }\end{array}\right.$ & "@context": \\
"@type": "Price Signal Request", & "@type": "Price Signal Response", \\
"name": "EnergyPlus", & "name": "EnergySurplus", \\
"cost": "\$3kWh" & "Power Quality": "95\%" \\
\} & \} \\
\hline
\end{tabular}

Figure 2. Open linked data for Scenario 1.

\section{Conclusions and Further Work}

Smart metering systems could be more important to the smart grid than today is. For this reason, it is necessary that the SMS and SG data can be open, linked, and with significance. JSON-LD can better understand all the concepts applied on power markets in Machine to Machine (M2M) communications.

\section{References}

[1] Wagner, A. et al. "Linked Data and Complex Event Processing for the Smart Energy Grid." LDSI@FIA (2010). http://ceur-ws.org/Vol-700/Paper10.pdf Proceedings of the Workshop on Linked Data in the Future Internet at the Future Internet Assembly. Ghent, Belgium, December 16-17, 2010.

[2] Villazón-Terrazas, B., Ortiz-Rodríguez, F., Tiwari, S. M., \& Shandilya, S. K. Knowledge Graphs and Semantic Web, 2020, https://doi.org/10.1007/978-3-030-65384-2.

[3] Smart Energy Aware System (SEAS), "Smart Meter Ontology", available at: https://ci.minesstetienne.fr/seas/SmartMeterOntology, last update: December 2020.

[4] Open Metering System (OMS), "The OMS Onthology", available at: https://sites.google.com/site/smartappliancesproject/ontologies/oms-ontology, last updated: December 2020.

[5] Smart Electric Power Alliance (SEPA), "Smart Grid Ontology", available at: https://sepapower.org/smart-grid-ontology/, last update: December 2020.

[6] National Institute of Standards and Technology (NIST), "Developing an Ontology for the Smart Grid (Discussion DRAFT)", available at:

https://www.nist.gov/system/files/documents/2018/11/08/draft_ontology_for_the_smart_grid_v2. pdf, last updated: December 2020. 
[7] Salameh K., Chbeir R., Camblong H. (2019) SSG: An Ontology-Based Information Model for Smart Grids. In Transactions on Large-Scale Data- and Knowledge-Centered Systems XL. Lecture Notes in Computer Science, vol 11360. Springer, Berlin, Heidelberg, doi: https://doi.org/10.1007/978-3-66258664-8_4

[8] D. Schachinger, W. Kastner and S. Gaida, "Ontology-based abstraction layer for smart grid interaction in building energy management systems," 2016 IEEE International Energy Conference (ENERGYCON), Leuven, 2016, pp. 1-6, doi: 10.1109/ENERGYCON.2016.7513991.

[9] J. L. Hippolyte et al., "Ontology-based demand-side flexibility management in smart grids using a multi-agent system," 2016 IEEE International Smart Cities Conference (ISC2), Trento, 2016, pp. 1-7, doi: $10.1109 /$ ISC2.2016.7580828.

[10] Gottschalk, M., et al., "The Smart Grid Architecture Model - SGAM. In: The Use Case and Smart Grid Architecture Model Approach. SpringerBriefs in Energy," Springer, Cham, doi: 10.1007/978-3319-49229-2_3.

[11] Vom Scheidt, F., et al., "Data analytics in the electricity sector - A quantitative and qualitative literature review, Energy and AI," Vol. 1, 2020, ISSN 2666-5468, doi: 10.1016/j.egyai.2020.100009.

[12] W. Medjroubi, et. al, "Open Data in Power Grid Modelling: New Approaches Towards Transparent Grid Models," in Energy Reports, Vol. 3, 2017, pp. 14-21, ISSN 2352-4847, doi: https://doi.org/10.1016/j.egyr.2016.12.001.

[13] S. Wilker, et al., "Smart grid architecture model standardization and the applicability of domain language specific modeling tools," 2017 IEEE 26th International Symposium on Industrial Electronics (ISIE), Edinburgh, UK, 2017, pp. 152-157, doi: 10.1109/ISIE.2017.8001239.

[14] N. Duan, C. Huang, C. -C. Sun and L. Min, "Smart Meters Enabling Voltage Monitoring and Control Functionalities: The Last-Mile Voltage Stability Issue," in IEEE Transactions on Industrial Informatics, doi: 10.1109/TII.2021.3062628.

[15] K. Tian, W. Sun, W. Liu and H. Song, "Coordinated RES and ESS planning framework considering financial incentives under centralized electricity market," in CSEE Journal of Power and Energy Systems, doi: 10.17775/CSEEJPES.2020.02400. 